Provided for non-commercial research and education use. Not for reproduction, distribution or commercial use.

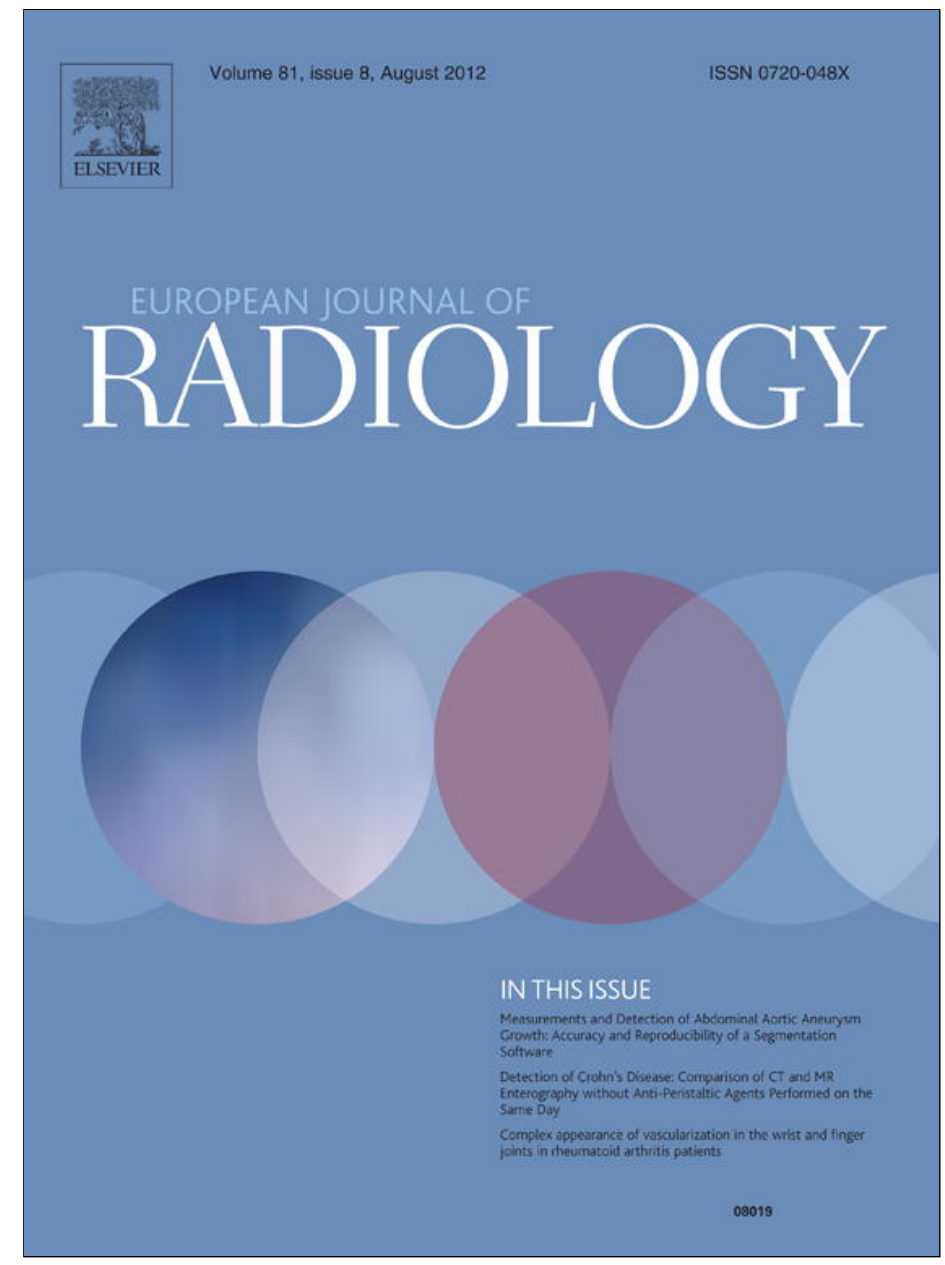

This article appeared in a journal published by Elsevier. The attached copy is furnished to the author for internal non-commercial research and education use, including for instruction at the authors institution and sharing with colleagues.

Other uses, including reproduction and distribution, or selling or licensing copies, or posting to personal, institutional or third party websites are prohibited.

In most cases authors are permitted to post their version of the article (e.g. in Word or Tex form) to their personal website or institutional repository. Authors requiring further information regarding Elsevier's archiving and manuscript policies are encouraged to visit:

http://www.elsevier.com/copyright 


\title{
Role of combined DWIBS/3D-CE-T1w whole-body MRI in tumor staging: Comparison with PET-CT
}

\author{
Guglielmo Manenti ${ }^{\mathrm{a}, *}$, Carmelo Cicciò ${ }^{\mathrm{a}, 1}$, Ettore Squillaci ${ }^{\mathrm{a}, 1}$, Lidia Strigari ${ }^{\mathrm{b}, 2}$, Ferdinando Calabriaa ${ }^{\mathrm{a}, 1}$, \\ Roberta Danieli $^{\mathrm{a}, 1}$, Orazio Schillaci ${ }^{\mathrm{a}, 1}$, Giovanni Simonetti ${ }^{\mathrm{a}, 1}$ \\ a Department of Diagnostic and Molecular Imaging, Interventional Radiology and Radiotherapy, University “Tor Vergata”, PTV Foundation - Policlinic “Tor Vergata”, Viale Oxford 81, \\ 00133 Rome, Italy \\ ${ }^{\mathrm{b}}$ Laboratory of Medical Physics and Expert Systems, Regina Elena National Cancer Institute, Via Elio Chianesi 53, 00144 Rome, Italy
}

\section{A R T I C L E I N F O}

\section{Article history:}

Received 25 April 2011

Received in revised form 7 August 2011

Accepted 8 August 2011

\section{Keywords:}

Whole-body magnetic resonance imaging

(WB-MRI)

Diffusion weighted imaging (DWI)

Diffusion-weighted whole-body imaging

with background body signal suppression

(DWIBS)

Positron emission tomography-computed

tomography (PET-CT)

Cancer staging

\begin{abstract}
A B S T R A C T
Objectives: To assess the diagnostic performance of whole-body magnetic resonance imaging (WB-MRI) by diffusion-weighted whole-body imaging with background body signal suppression (DWIBS) in malignant tumor detection and the potential diagnostic advantages in generating fused DWIBS/3D-contrast enhanced T1w (3D-CE-T1w) images.

Methods: 45 cancer patients underwent 18F-FDG PET-CT and WB-MRI for staging purpose. Fused DWIBS/3D-CE T1w images were generated off-line. 3D-CE-T1w, DWIBS images alone and fused with 3D-CE T1w were compared by two readers groups for detection of primary diseases and local/distant metastases. Diagnostic performance between the three WB-MRI data sets was assessed using receiver operating characteristic (ROC) curve analysis. Imaging exams and histopathological results were used as standard of references.

Results: Areas under the ROC curves of DWIBS vs. 3D-CE-T1w vs. both sequences in fused fashion were $0.97,0.978$, and 1.00 , respectively. The diagnostic performance in tumor detection of fused DWIBS/3DCE-T1w images were statistically superior to DWIBS $(p<0.001)$ and 3D-CE-T1w $(p \leq 0.002)$; while the difference between DWIBS and 3D-CE-T1w did not show statistical significance difference. Detection rates of malignancy did not differ between WB-MRI with DWIBS and 18F-FDG PET-CT.

Conclusion: WB-MRI with DWIBS is to be considered as alternative tool to conventional whole-body methods for tumor staging and during follow-up in cancer patients.
\end{abstract}

(c) 2011 Elsevier Ireland Ltd. All rights reserved.

\section{Introduction}

Local and distant tumor staging have a pivotal role for therapeutic option and prognosis of oncologic diseases. As tumor spread may involve different anatomical regions, accurate whole-body evaluation requires common multimodality approach, including conventional radiography, ultrasonography (US), computed tomography $(\mathrm{CT})$, scintigraphy and positron emission tomography (PET).

Over the last decade, positron emission tomography (PET) with 2-deoxy-2 [F] fluoro-D-glucose (FDG) in combination with CT

\footnotetext{
* Corresponding author. Tel.: +3906 20902401; fax: +390620902404. E-mail addresses: guggi@tiscali.it (G. Manenti), carmeciccio@libero.it (C. Cicciò), ettoresquillaci@tiscali.it (E. Squillaci), strigari@ifo.it (L. Strigari), ferdinandocalabria@hotmail.it (F. Calabria), roberta.danieli@ptvonline.it (R. Danieli), orazio.schillaci@uniroma2.it (O.Schillaci),

giovanni.simonetti@uniroma2.it (G. Simonetti).

1 Tel.: +3906 20902401; fax: +390620902404.

2 Tel.: +3906 52661; fax: +390652666236.
}

(PET-CT) has become an important non-invasive imaging modality for the preoperative staging of various tumors. Metabolic information, provided with PET exam, is combined to anatomical data of CT scan, resulting in higher diagnostic accuracy compared with the two imaging modalities alone and with both imaging modalities viewed side-by-side [1].

Whole-body magnetic resonance imaging (WB-MRI), with its lack of ionizing radiation, but high contrast and spatial resolution, is a promising whole body imaging tool for detection and staging of various neoplastic diseases, especially for tumors frequently metastasizing to the abdominal organs, bone, brain (e.g. lung, breast, colorectal, prostate cancers) or for haematologic diseases with nodal or bone marrow involvement [2].

Up-to-date, WB-MRI provided mainly morphological information on tumor spread; however, the lack of functional informations has been overcome by the introduction in clinical practice of diffusion whole body imaging. Diffusion-weighted whole-body imaging with background body signal suppression (DWIBS) is a newly diagnostic sequence, introduced in recent years in WB-MRI protocols [3]. DWIBS provides functional data about tumor cellularity and has 
Table 1

Patient characteristics.

\begin{tabular}{lr}
\hline Male & 24 \\
Female & 21 \\
Age (range) & 66 (48) \\
Tumor histopatology & 10 \\
Colo-rectal cancer (CRC) & 7 \\
Lung cancer (LC) & 9 \\
Hodgkin lymphoma (HL) & 10 \\
Multiple myeloma (MM) & 9 \\
Breast cancer (BC) & \\
Diagnosed lesions & 3 \\
Adrenal metastases & 39 \\
Bone lesions in multiple myeloma patients & 32 \\
Bone metastases & 5 \\
Brain metastases & 4 \\
Breast cancer & 29 \\
Liver metastases & 3 \\
Colo-rectal cancer & 54 \\
Lymph nodes metastases & 22 \\
Lung metastases & 5 \\
Extra-medullary disease in multiple myeloma patients & 6 \\
Bulky mass & \\
Other findings & \\
Pancreatic cysts & 5 \\
Liver cysts & 29 \\
Hepatic haemangiomas & 21 \\
Rymp nodes & 26 \\
Vertebral haemangiomas & 32 \\
Osteoporotic vertebrae fracture & 13 \\
Ovarian cysts & 1 \\
Splenic haemangioma & 7 \\
\hline & 1 \\
\hline
\end{tabular}

been found to be of complementary value to morphological imaging studies when assessing lymph node involvement or distant metastasis [4]. With DWIBS, tumor sites may be detected throughout the entire body with high contrast resolution; however, exact localization of lesions with DWIBS may be less accurate due to its relatively low spatial resolution and lack of anatomical reference because most normal anatomic structures signal is suppressed $[5,6]$.

These drawbacks may be overcome by combining DWIBS with 3D fat suppressed contrast enhanced $\mathrm{T} 1$ weighted MR imaging (3D-CE-T1W; e.g. T1-weighted high-resolution isotropic volume examination, THRIVE) to provide anatomical information and to differentiate malignant from normal tissue.

The aim of our study is to retrospectively assess the diagnostic performance of DWIBS, WB-MRI and fused DWIBS/3D-CE-T1w images for malignant tumor detection and to evaluate if there is an additional value in generating fusion images of DWIBS and 3D contrast-enhanced images.

\section{Materials and methods}

\subsection{Subjects}

This study was performed after approval of the local institutional review board. Written informed consent was obtained from all participants. A total of 45 consecutive patients ( 24 male and 21 female; mean age of 66 years; range 48-79) with diagnosis of malignant disease were included in the study. Primary tumors included 7 lung carcinoma (LC), 9 Hodgkin's lymphoma (HL), 10 colo-rectal cancers (CRC), 10 multiple myeloma (MM), 9 breast cancer (BC) (Table 1). All patients were submitted to 18F-FDG-PETCT for staging purpose, according to our institutional guidelines. Histopathological specimens were used as standard of reference for primary tumors in all cases. Imaging follow-up at 12 months and histopathological results, where available, were used as a gold standard.

\subsection{MR imaging}

WB-MRI was performed with a 3.0 Tesla MR scanner (Achieva, Philips Healthcare, $80 \mathrm{mT} / \mathrm{m}$ maximum amplitude, $0.16 \mathrm{~ms}$ minimal rise time, $200 \mathrm{~T} / \mathrm{m} / \mathrm{s}$ maximum slew rate) with patient in supine feet-first position, covering the entire body as a matrix with a maximal longitudinal field of view (FOV) of $200 \mathrm{~cm}$, in combination with automated table movement. Whole-body images were obtained with multiple stacks acquisitions ( 7 or 8 overlapped stacks depending on body height, as follow: head/neck, thorax, abdomen, pelvis, thighs, knees and calves), using Q-body coil for signal receiving and transmitting. Our standard whole-body MR imaging protocol consisted of unenhanced T1-weighted and T2weighted turbo field-echo (TFE), DWIBS sequences and 3D-CE-T1W sequences (THRIVE) (parameters in Table 2 ).

DWIBS images were acquired using single-shot echo-planar imaging (EPI) sequences with a short inversion time inversion recovery (STIR) pre-pulse for fat suppression. 60 slices each stack ( $n$. 4: head/neck; thorax; abdomen; pelvis) were obtained using free-breathing technique on axial plane; motion probing gradients (MPGs) in three orthogonal axes were applied for two $b$ value 0 and $1000 \mathrm{~s} / \mathrm{mm}^{2}$, with 6 signal sampling average. Gadolinium-enhanced isotropic three-dimensional T1-weighted high-resolution isotropic volume acquisition with fat suppression (3D-CE-T1w; THRIVE) imaging was performed on axial plane before and after administration of a paramagnetic contrast agent $(0.2 \mathrm{mmol} / \mathrm{kg}$ gadopentetate dimeglumine, Magnevist; Bayer-Schering, Berlin, Germany), injected into the antecubital vein ( $3 \mathrm{ml} / \mathrm{s}$ flow rate) followed by $20 \mathrm{ml}$ saline solution using an automated injector (Spectris MR, Medrad Europe). After i.v. administration of a paramagnetic contrast agent $(0.1 \mathrm{mmol} / \mathrm{kg}$ of gadolinium), 3D data sets of the abdomen were acquired in axial plane in the early arterial and portal venous phases. Subsequently, the rolling table platform was moved to the skull, thorax, abdomen, pelvis and femur to acquire data sets in coronal plane during equilibrium contrast phase. Total acquisition time for whole-body MRI was less than $60 \mathrm{~min}$.

\subsection{F-FDG-PET-CT}

All examinations were performed with an integrated PET/CT scanner, Discovery ST (GE, General Electric Medical Systems, Milwaukee, WI, USA). The system combines a high speed ultra 16detector-row CT scanner with a PET scanner equipped with 10080 bismuth germinate crystals arranged in 24 rings. Patients were required to fast for at least $6 \mathrm{~h}$ prior to the examination

A baseline CT scan with low tube current $(80 \mathrm{~mA})$ was then acquired to correct the attenuation for the PET study. The following scan parameters were used: $140 \mathrm{kV}$ to obtain good quality images at the level of the shoulder and pelvic girdles; $80 \mathrm{~mA}$; FOV 420-500 $\mathrm{mm}$; CT slice thickness $3.75 \mathrm{~mm}$ (retrospectively reconstructed to $1.25 \mathrm{~mm}$ ) to approximate the width of the PET section; slice interval $3.27 \mathrm{~mm}$, to coincide with the spacing between the PET sections; scan speed $<1 \mathrm{~s}$ per revolution. Once the CT scan was completed, the PET scan was performed with a $2 \mathrm{D}$ technique in the caudo-cranial direction from the proximal third of the femurs to the head; five to six bed positions were acquired, with duration time of $4 \mathrm{~min}$ per bed. Images were reconstructed using a standard iterative algorithm [ordered subsets expectation maximisation (OSEM)], with a mean duration of 20-24 min for the PET examination. PET acquisition was immediately followed by CT examination performed with the intravenous administration of $100-120 \mathrm{ml}$ of non-ionic iodinated contrast medium with injection rate of $2-3 \mathrm{ml} / \mathrm{s}$ (Iomeron $350 \mathrm{mgI} / \mathrm{ml}$, Bracco, Milan, Italy). Two data sets were acquired: the first included the upper abdomen with a delay of $30 \mathrm{~s}$ from the beginning of the contrast injection, and the 
Table 2

Technical parameters of applied sequences (TFE, turbo field echo; FOV, field of view; FFE, fast field echo; TR, repetition time; TE, echo time; thk, slice thickness; NSA, number of signal average; DWIBS, diffusion weighted whole-body imaging with background body signal suppression; DWI, diffusion weighted imaging; EPI, echo-planar imaging; STIR, short tau inversion recovery).

\begin{tabular}{|c|c|c|c|c|}
\hline & T1w & $\mathrm{T} 2 \mathrm{w}$ & 3D-CE-T1w (THRIVE) & DWIBS \\
\hline Sequences & TFE & TFE & FFE & DWI-EPI-STIR \\
\hline Stacks & $7-8$ & $7-8$ & 4-5 (with 2 dyn scans at upper abdomen) & $5-6$ stacks \\
\hline FOV & $470-500$ & $470-500$ & 395 & $375-390$ \\
\hline Plane & Coronal & Coronal & $\begin{array}{l}\text { Axial plane (upper abdomen) Coronal plane } \\
\text { (skull-thorax-upper abdomen-pelvis) }\end{array}$ & Axial (reformatted on coronal plane) \\
\hline TR/TE/flip angle & Shortest $/ 7.6 / 100^{\circ}$ & $3200 / 120 / 120^{\circ}$ & $1.8 / 2.4 / 10^{\circ}$ & Shortest $/ 260^{\circ}$ \\
\hline$b$ value $\left(\mathrm{s} / \mathrm{mm}^{2}\right)$ & - & - & - & $0-1000$ \\
\hline n.Sl/thk/intersl gap & $30-40 / 6 \mathrm{~mm} / 2 \mathrm{~mm}$ & $30-40 / 6 \mathrm{~mm} / 2 \mathrm{~mm}$ & $100-110 / 4 \mathrm{~mm} /-2 \mathrm{~mm}$ & $30-40 / 6 \mathrm{~mm} / 2 \mathrm{~mm}$ \\
\hline Matrix/voxel size & 320 & 320 & $154 \times 256 / 1.13 \mathrm{~mm}^{3}$ & 256 \\
\hline $\mathrm{NSA} /$ scan duration & $2 / 9 \min$ & $2 / 13 \mathrm{~min}$ & $2 / 1: 14$ & $6 / 12 \min$ \\
\hline
\end{tabular}

second extended from the neck to the pubic symphysis with a delay of $60-80 \mathrm{~s}$. An additional scan was performed in the late phase to correctly characterize lesions with suspected features at previous scans. Lastly, after having the patients placed their arms on the sides, axial scans of the head were acquired.

The following scan parameters were used: $120-140 \mathrm{kV}$, automatic tube current (limit 330-350 mA), slice thickness $3.75 \mathrm{~mm}$ (retrospectively reconstructed to $1.25 \mathrm{~mm}$ ), acquisition mode 27.50/1.375:1, gantry rotation speed $0.6 \mathrm{~s}$, FOV large, and matrix $512 \times 512$. The average overall duration of the entire PET/CT acquisition was $40 \mathrm{~min}$.

\subsection{Image interpretation and analysis}

All WB-MR images were transferred and analyzed off-line onto a workstation (View Forum, Philips Health-Care). Two radiologists, fully blinded to the results of previous or current diagnostic imaging modalities, independently analyzed, during three different reading sessions, the following WB-MRI data sets: session 1, DWIBS images; session 2, 3D-CE-T1W images; session 3, fused DWIBS/3D-CE-T1W images. Within each reading session, the two readers did not have a time limit for image analysis. The duration of each reading session was approximately $15-20 \mathrm{~min}$ and the time period that separated the three reading sessions was approximately 5 days. The images of each patient were analyzed by the two readers in a random order and mixed with control group subjects.

To assess the accuracy of each sequence in detection of pathological lesions at a given time point, we analyzed the results twice: first, categorizing the equivocal findings as suggestive for malignancy and after that as suggestive for benign.

Image interpretation regarded the detection of primary disease and assessment of local/distant metastasis. During single analysis, DWIBS series were evaluated on axial and coronal plane reformatted from the axial images using maximum intensity projection (MIP). The images were displayed in three-dimensional cine mode in PET-like fashion with inversion of gray-scale.

3D-CE-T1W series were evaluated on axial plane and after reformation using MPR (multiplanar reformation) algorithms. Freeware DICOM software (OsiriX medical imaging software, OsiriX, Atlanta, GA, USA) was used to obtain fused images on coronal or axial plane between DWIBS (displayed using "hot-iron" colour map) and 3DCE-T1W images (as shown in Fig. 1).

On visual analysis of MR images, each site of abnormal and/or focal increase of DWIBS intensity not from normal anatomic structure or with unexpected increase in signal intensity after administration of paramagnetic contrast agent on 3D-CE-T1W images was considered as malignant, benign or equivocal for primary/distant disease. DWIBS was evaluated only visually and in comparison with signal intensity of surrounding tissue. In particular lymph nodes were considered pathologically involved according to morphologic criteria (short axis diameter $>10 \mathrm{~mm}$; long axis-to short axis ratio $>1$ ) and/or by the evidence of increase in signal intensity on DWIBS images in comparison with spinal cord. Quantitative analysis of ADC value (ADC, apparent diffusion coefficient) was not performed in this study.

When the high signal on DWIBS images correspond to normal structure or no corresponding abnormality was demonstrated at 3D-CE-T1W, the high signal was considered false positive and identified as non malignant. Discrepancies were resolved by consensus, as well as using data sets provided by T1W-TFE and T2W-TFE sequences enclosed in our imaging protocol.

18F-FDG-PET-CT images were processed on an AdvantageWindows 4.4 workstation (GE, General Electric Medical Systems) individually for PET and CT examination and with PET/CT fusion software. Areas with focal increased of 18F-FDG-uptake that exceeded the accumulation of tracer in the anatomical region or with abnormal density or contrast enhancement after administration of iodinate material contrast, were considered as malignant. The analysis was conducted on the images corrected for attenuation and in the case of doubt also on the uncorrected images. Acquisition data of all CT examinations were processed and retrospectively reconstructed with the following parameters: slice thickness $1.25 \mathrm{~mm}$ and interval $3.27 \mathrm{~mm}$ to coincide with the spacing between the PET sections. Images obtained with CT and PET were independently reviewed in consensus by a radiologist and a nuclear physician.

18F-FDG-PET-CT imaging follow-up for at least 12 months and histopathological results, if available, were used to get a final diagnosis of the equivocal lesions.

\subsection{Statistical analysis}

Sensitivity, specificity, diagnostic accuracy, positive predictive value (PPV), and negative predictive value (NPV) were calculated for each imaging dataset, for all readers combined.

To assess differences in diagnostic performance between the three WB-MRI imaging datasets (DWIBS, 3D-CE-T1w and both sequences in fused fashion) in the detection of tumor spread on lesion-by-lesion basis, receiver operating characteristic (ROC) analysis was performed.

The areas under the ROC curves (AUCs) were compared for each of the three datasets from all readers combined and between the readers for each dataset. The ROC curves were compared with an area test. The data were considered ordinal and were correlated. A two-tailed $p$-value was used to compare AUCs of DWIBS with THRIVE, while a one-tailed $p$-value was used to compare AUCs of DWIBS or THRIVE with both sequences. This decision was based on a prospective determination that a combined modality should be equal or superior to its components.

In every statistical analysis, a $p$-value of less than 0.05 was considered to indicate a statistically significant difference. 
Table 3

Assessment of focal lesion by THRIVE, DWIBS and fused DWIBS/3D-CE-T1w images.

\begin{tabular}{|c|c|c|c|c|c|}
\hline & \multicolumn{5}{|c|}{ Diagnostic perfomance ${ }^{a}$} \\
\hline & Sensitivity (\%) & Specificity (\%) & PPV (\%) & NPV (\%) & Accuracy (\%) \\
\hline THRIVE & $96(96)$ & $100(97)$ & $100(100)$ & $92(94)$ & $97(96)$ \\
\hline DWIBS & $94(100)$ & $100(70)$ & $100(83)$ & $92(100)$ & $96(88)$ \\
\hline Fused DWIBS/3D-CE-T1w & $100(100)$ & $100(98)$ & $100(99)$ & $100(100)$ & $100(99)$ \\
\hline
\end{tabular}

a Analysis considering equivocal (E) findings as benign/negative $(\mathrm{B} / \mathrm{N})$; in parentheses, diagnostics performance considering equivocal (E) results as positive for malignancy (M).

Table 4

AUCs of the readers for each data sets (data in parentheses are confidence intervals at 95\%).

\begin{tabular}{llll}
\hline & DWIBS $(\mathrm{CI})$ & 3D-CE-T1w $(\mathrm{CI})$ & DWIBS/3D-CE-T1w (CI) \\
\hline Readers 1 & $0.958(0.928-0.978)$ & $0.975(0.950-0.990)$ & $1.000(0.987-1.000)$ \\
Readers 2 & $0.953(0.922-0.974)$ & $0.973(0.947-0.988)$ & $1.000(0.987-1.000)$ \\
All & $0.970(0.944-0.986)$ & $0.978(0.954-0.991)$ & $1.00(0.988-1.000)$ \\
$p$-Value 1 vs. 2 & 0.726 & 0.808 & 1.000 \\
\hline
\end{tabular}

\section{Results}

A total number of 202 malignant lesions were spotted in 45 patients (Table 1). The diagnosis of primary tumors was based upon biopsy or histopathology after surgery in all patients. Validation of suspected lymph nodes or distant metastasis was achieved by surgery $(n=68)$, complementary imaging methods $(n=78)($ e.g. bone scintigraphy, MRI, PET-CT or CT), and/or clinical/radiological follow-up $(n=49)$ for at least 12 months. Details of patients and pathological findings are shown in Table 2.

In the evaluation of 202 true malignant lesions detected at PETCT exam, reader 1 identified 183 and 19 lesions as malignant and equivocal on DWIBS images and 191, 2 and 9 lesions as malignant, equivocal and benign on 3D-CE-T1W images; reader 2 considered 185, 17 lesions as malignant and equivocal on DWIBS images and 192, 1 and 9 lesions as malignant, equivocal and benign on 3D-CE-T1W, respectively. In this group, the two readers showed agreement in the evaluation of 190 lesions (178 malignant and 12 equivocal) on DWIBS images and 199 lesions (190 malignant and 9 benign) on 3D-CE-T1W images. 12 and 3 disagreement targets (equivocal/malignant) at DWIBS and 3D-CE-T1W were considered malignant after consensus reading. Each reader independently considered 12 (9 lymph nodes; 3 liver metastasis) and 9 (all lymphnodes) malignant lesions as equivocal and benign at DWIBS and 3D-CE-T1W, respectively.

The positive predictive value, negative predictive value, sensitivity, and specificity are summarised in Table 3.

In the assessment of lesions, areas under the ROC curves of DWIBS vs. 3D-CE-T1W vs. both sequences in fused fashion were $0.97,0.978$, and 1.00 , respectively (Table 4 ). The ROC curves
Table 5

$p$-Values of pairwise comparison of ROC curves of different datasets evaluated by two readers.

\begin{tabular}{llll}
\hline & & & \\
\cline { 2 - 4 } & DWIBS vs. & DWIBS vs. & 3D-CE-T1w vs. \\
& 3D-CE-T1w & DWIBS/3D-CE- & DWIBS/3D-CE- \\
& & T1w & T1w \\
\hline Readers 1 & 0.130 & $<0.001$ & 0.001 \\
Readers 2 & 0.154 & $<0.001$ & 0.002 \\
\hline
\end{tabular}

are shown in Fig. 2. In the assessment of individual lesions, fused DWIBS/3D-CE-T1W images were statistically superior to DWIBS $(p<0.001)$ and 3D-CE-T1W $(p \leq 0.002)$; while the difference between DWIBS and 3D-CE-T1W did not reach statistical significance in our population $(p=$ N.S.) (Table 5).

Primary tumors and distant metastasis detection rates did not differ between WB-MRI with DWIBS and PET-CT (Figs. 3 and 4). In lesion-by-lesion analysis, diagnostic performance of DWIBS for the detection of lymph node metastasis, expressed in terms of sensitivity and specificity, ranged from 83 to $100 \%$ and $80-100 \%$, due to the high number of equivocal lymph-node without focal impeded of DWI signal intensity; using anatomical information provided by 3D-CE-T1W images, diagnostic performance of DWIBS yields a sensitivity and specificity similar to the gold standard used, upstaging 9 suspected loco-regional lymph-node as malignant. Normal size and faded contrast enhancement were demonstrated for corresponding lesion at 3D-CE-T1W. Malignant status was confirmed at histopatogical specimen after surgery treatment of primary tumor.
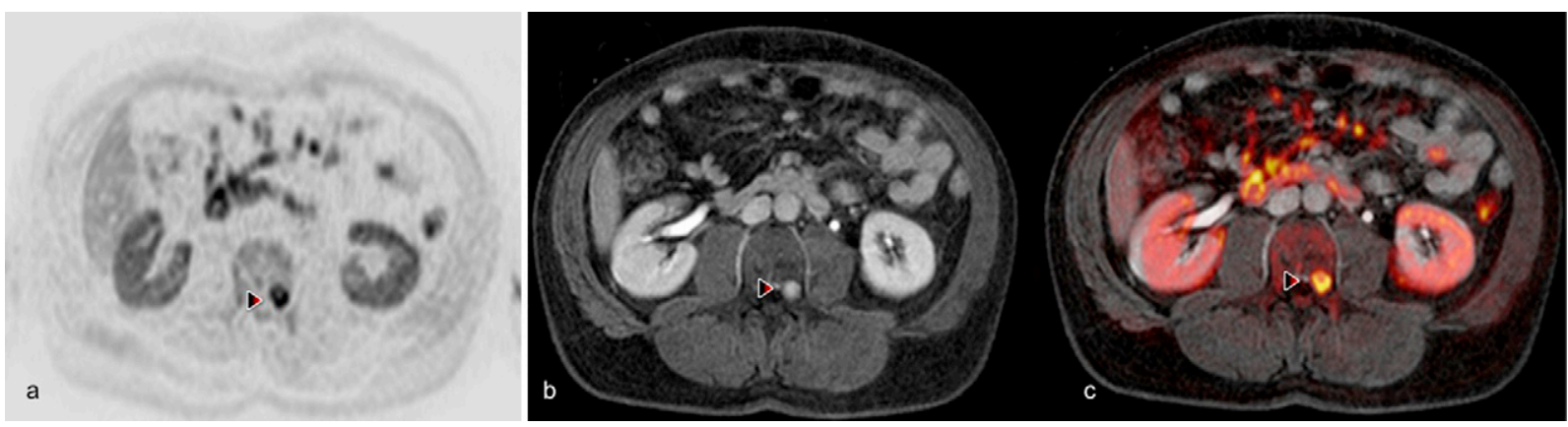

Fig. 1. 62-Years-old female with bone metastatic disease from breast cancer. (a) Axial DWIBS images shows focal area (arrowhead) with impeded signal intensity suspected for lumbar vertebra metastasis; (b) 3D-CE-T1w image on axial plane at the same level show focal area (arrowhead) with pathological contrast enhancement at the same level; (c) fusion DWIBS/3D-CE-T1w clarifies that abnormal signal on DWIBS correspond to metastatic lesion located at left pedicles of a lumbar vertebra (arrowhead). 
a

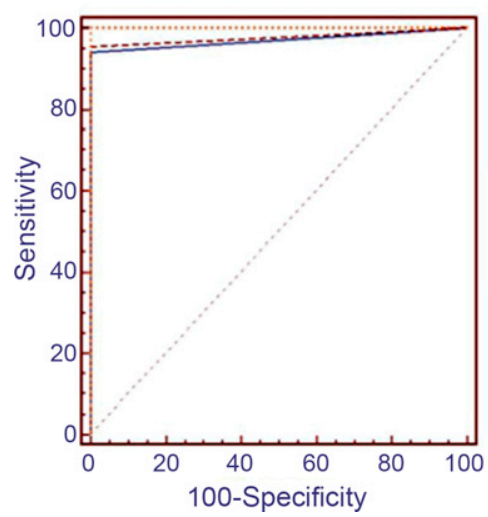

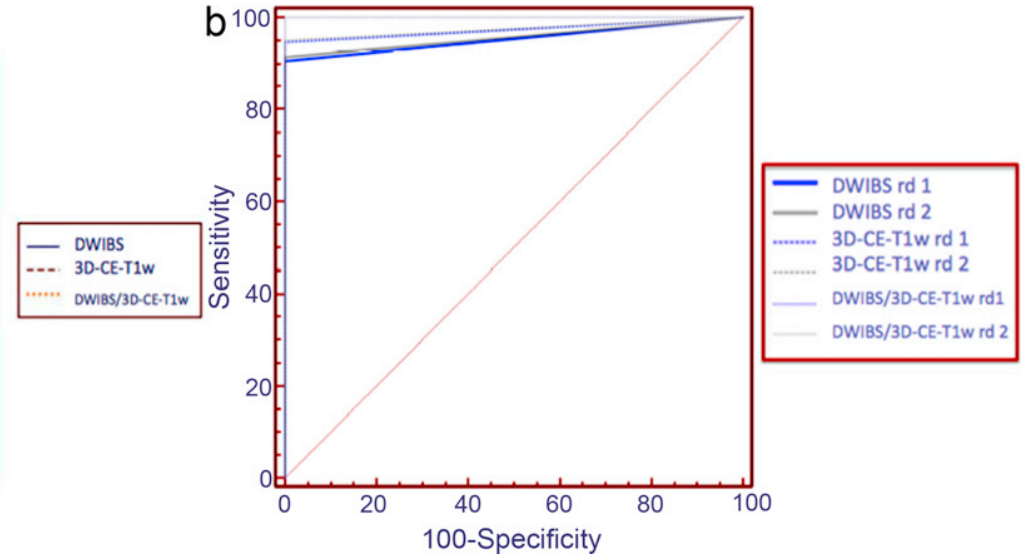

100-Specificity

Fig. 2. ROC curves for each data sets in the detection of malignant lesions for all reader combined (a) and reader 1 (rd1) and reader 2 (rd2) (b).
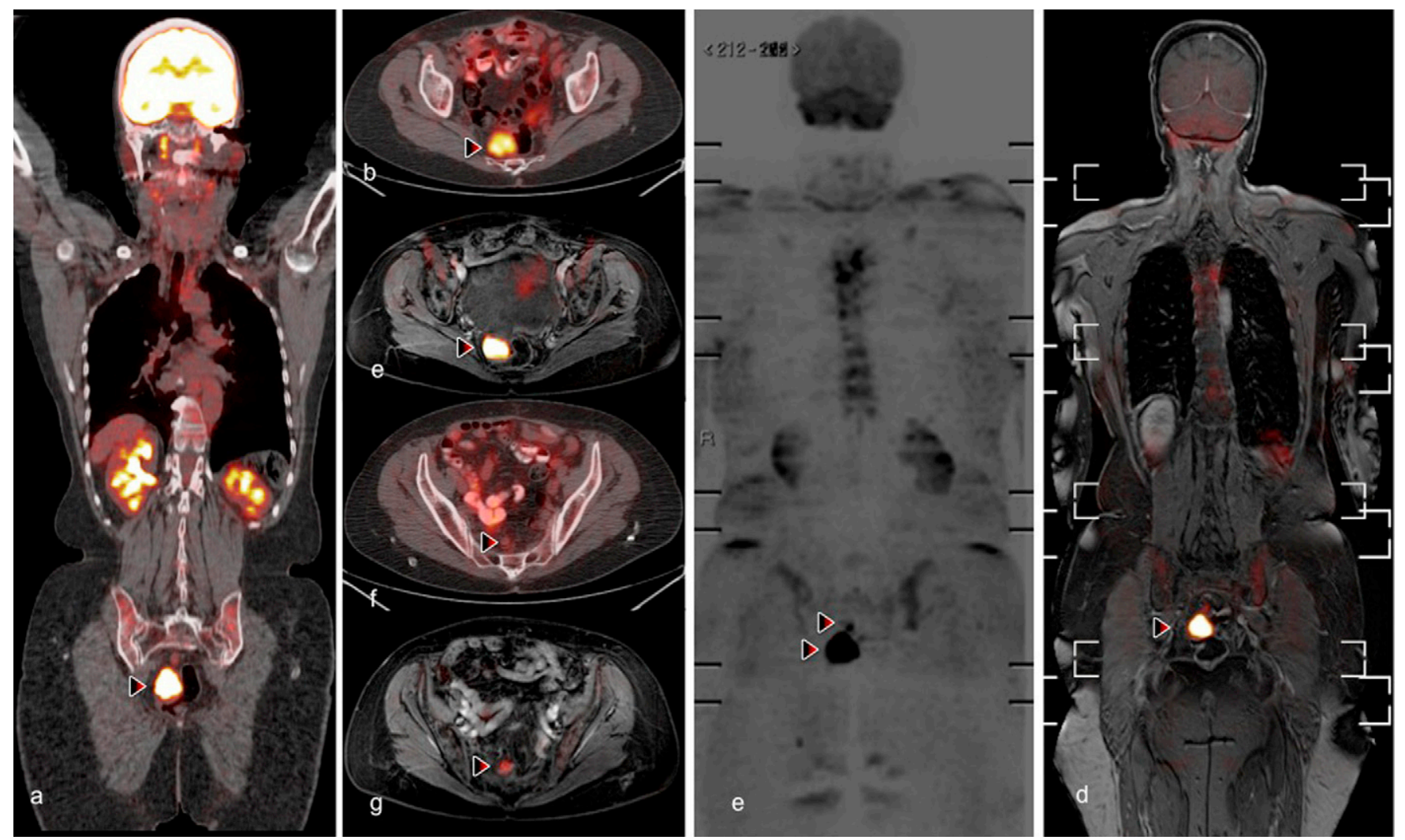

Fig. 3. 55 years-old male with recent diagnosis of colo-rectal adeno-carcinoma. High correlation between 18F-FDG PET-CT and DWIBS/3D-CE-T1w fused images in preoperative imaging staging of colo-rectal cancer and distant lymph-node metastases. (a, b) 18F-FDG PET-CT detected, on coronal (a) and axial (b) plane, the primitive tumor as high metabolic lesion in the lateral wall of the sigma; (c, d, e) DWIBS alone (c) and fused with 3D-CE-T1w on coronal (d) and axial plane (e), identify at the same localizzation, colo-rectal tumor as mass with high intense restriction of diffusion; (f, g) one metastatic lymph-node in pre-sacral chain was detected as lesion with high tracer uptake on axial FDG-PET-CT fused image (d) and with restricted diffusion on DWIBS alone ad combined with 3D-CE-T1w.

No disagreement was found in the readings of fused images for the same lesions between the two readers. In particular, the AUC of ROC curve for 3D-CE-T1W fused with DWIBS was significant higher than those of DWIBS $(p<0.001)$ and 3D-CE-T1W $(p<0.002)$; not significant difference was found in AUCs of corresponding sequences between both readers.

DWIBS images quality was rated as good in 40 of 45 patients. Susceptibility artefacts were observed in three patients, probably due to the use of high strength fields (3.0Tesla); in two patients misregistration artefacts were demonstrated at the base of the lungs. The patient was required to keep quiet breath-hold during 3D-CE-T1W images while quite free breathing was maintained during DWIBS scan. Although some misregistration artefacts were observed in fused images, these artefacts did not significantly hamper diagnostic content of images acquired.

\section{Discussion}

Functional techniques are important tools for the detection and characterization of malignant lesions as well as for evaluation of response to treatment. Up to date, 18F-FDG PET alone or combined with CT has been an essential method for detection and staging of a wide number of malignancies. Potential drawbacks that may limit its use in oncology are related to some pitfalls in the interpretation 

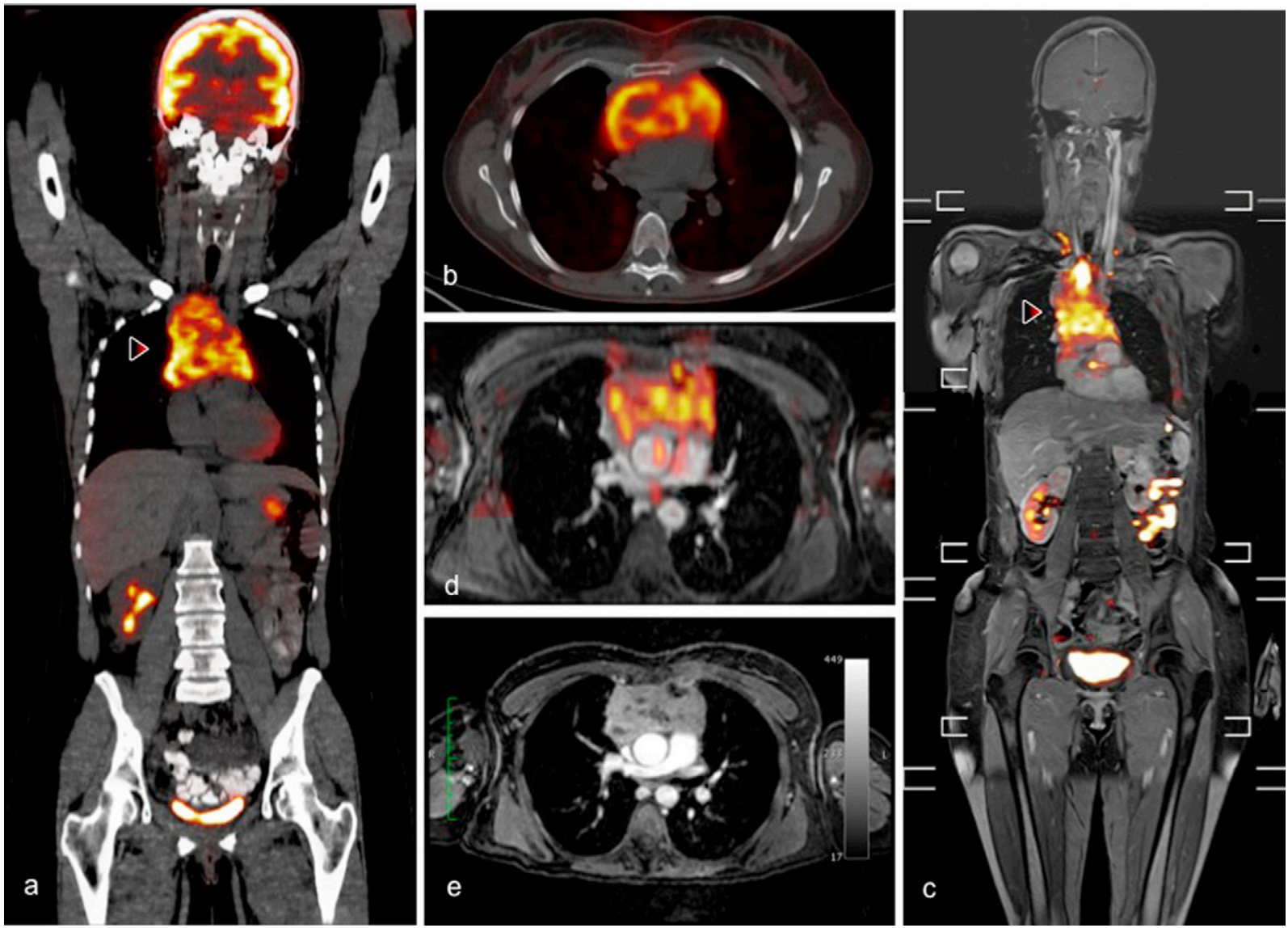

Fig. 4. 45 years-old female with diagnosis of Hodgkin's Lymphoma. (a, b) Fused 18F-FDG-PET-CT on coronal (a) and axial (b) plane shows bulky mediastinic mass with pathologic FDG-uptake; (c, d) DWIBS images in coronal (c) and axial plane (d), fused with 3D-CE-T1w, demonstrate high signal intensity in corresponding mediastinic lesion; (e) the mass show disomogeneous contrast enhancement on axial 3D-CE-T1 image.

of PET or CT scans or both modalities combined. Although PET has high sensitive in the detection of a huge number of tumor lesions, there are a small number of malignancies with poor FDGuptake, such as bronchoalveolar carcinoma, neuroendocrin tumors, colonic mucinous adenocarcinoma, prostate carcinoma and carcinoid tumor. On the contrary, potential false positive FDG-uptake may be observed in association with inflammatory conditions (abscess, granulomatous disease, atherosclerosis) or with benign tumors (colonic adenoma, uterine fibroids) [7].

Although the fusion of PET images with a CT scan may improve lesions detection and localization, this combined morphofunctional imaging modality may provide some interpretative difficulties. CT map for correction of attenuation may generate artefacts that can affect the PET images. For instance, the use of contrast medium and the presence of metallic implants can be associated with false positive focal radiotracer uptake. Furthermore, misregistration artefacts related to patients breathing, bowel motions, may result in wrong organ superimposition of tracer uptake. To note, the potential risk related to radiation exposure, especially during long imaging follow-up, the limited availability and costs.

With the recent technological developments, new functional imaging tools became available for daily application in association with well-established metabolic and functional imaging methods (e.g. PET, Scintigraphy).

DWI is a functional magnetic resonance technique, sensitive to the microscopic mobility of water (Brownian motion), due to its thermal movement; it is a completely non-invasive sequence, not requiring the administration of contrast medium and derives its image features from differences in the motion of water molecules trough intra- and extra-cellular spaces. Although DWI is a wellestablished technique used in brain exam, due to high sensitivity for stroke event, the increased availability of high-strength field scanner, generating high performance gradient combined to the use of fast imaging sequences, like echo-planar imaging (EPI), parallel imaging technique and multi-channel coil system, allow DWI to be used in daily imaging protocols, also for non-neurological applications.

Atypical signal intensity in DWI images is considered early imaging biomarker of tissue abnormalities (e.g. ischemia, malignant disease) and, combined with morphological data provided by conventional MR imaging, may be used for tissue analysis. In particular, by the evidence of its capability in differentiating benign from malignant lesions, DWI is frequently added into oncological imaging protocols for primary tumor detection, evaluation of lymph-node status and distant metastatic involvement, monitoring of response to treatment and distinguishing post-therapeutic response vs. residual viable tumor [8,9]. DWIBS is a particular DWI (Diffusion-weighed Imaging) technique, firstly proposed by Takahara et al. [10], providing functional information from entire body (whole-body) during free breathing, in high SNR images, displayed in PET-like fashion.

Whole-body MRI (WB-MRI) has been introduced in recent years as imaging method with promising clinical impact for tumor staging and screening [11].

Only limited experiences have compared the diagnostic performance of PET-CT vs. WB-MRI in oncological series, commonly concerning metastasis screening or tumor staging. Furthermore, the larger series reveal a diagnostic performance of WB-MRI 

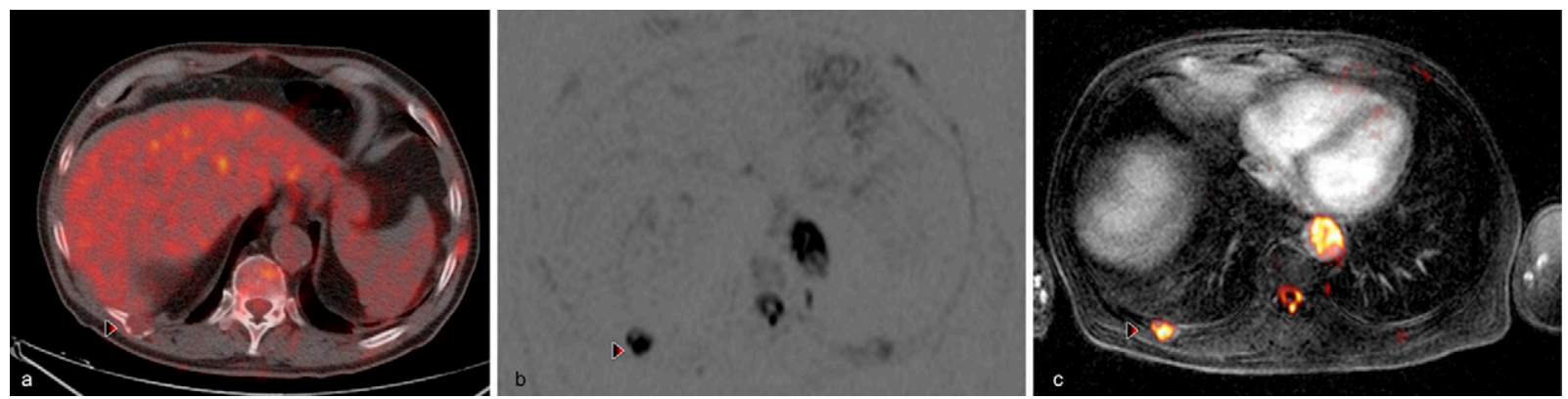

Fig. 5. 45 years-old patients with diagnosis of multiple myeloma. (a) 18F-FDG PET-CT show no FDG-avid malignant soft tissue, determining bone lytic lesion on posterior arch of a right rib; (b, c) at DWIBS alone (a) and fused with 3D-CE-T1w (c) this lesion induce bone deformity at the same rib levels, showing focal restricted signal of diffusion.
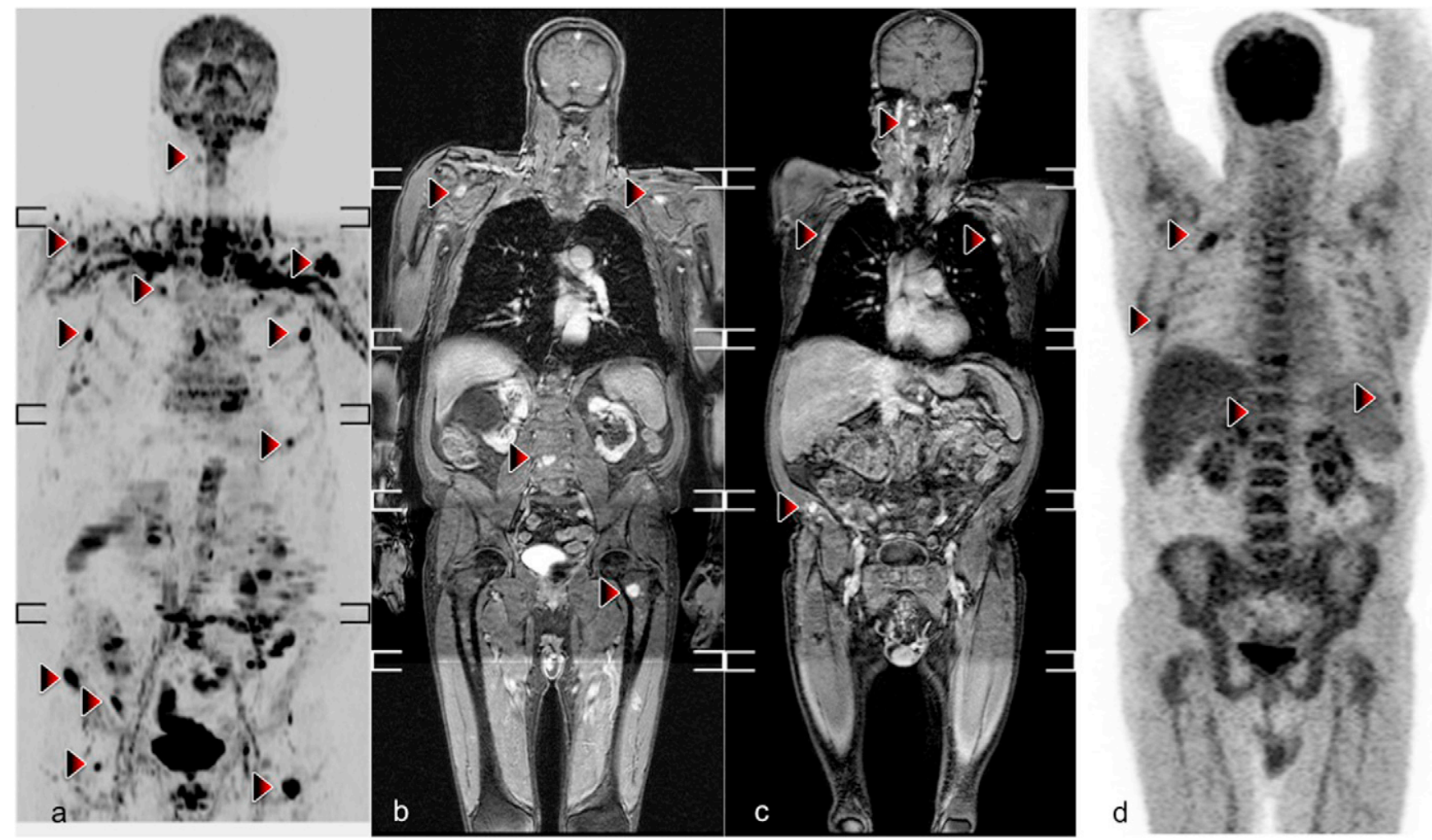

Fig. 6. Comparison WB-MRI with DWIBS and 18F-FDG PET-CT in staging newly diagnosed breast cancer in 47 years-old female. (a) Coronal $18 \mathrm{~F}-\mathrm{FDG}$ PET-CT (a) and axial 18F-FDG PET-CT ( $\left.a^{\prime}\right)$ demonstrate high tracer uptake at nodular lesion (arrowhead) in left breast, suggestive for primary breast cancer; (b) Corresponding coronal DWIBS, displayed with gray scale inversion, shows focal area with impeded signal intensity (b) on left breast at nodular lesion with significant c.e. (b') after administration of Gd-DTPA e.v.; (c) coronal DWIBS shows two focal areas (c; black encircled) with restricted diffusion at left axillar region, suspected (only for intensity signal characteristic) for malignant involved lymph nodes. DWIBS is limited in the discrimination between benign from malignant nodes using features or size criteria. At 3D-CE-T1w images ( $c^{\prime}$ ) show normal sized lymph-node (about $13 \mathrm{~mm}$; only one shown) with lipomatous center and non significant c.e. after Gd-DTPA, suggestive for benign features. (d) Coronal maximum intensity projection 18F-FDG PET-CT do not show significant tracer uptake at left axillar at lymph-node ( $\left.\mathrm{c}^{\prime}\right)$ confirm its benign nature.

difficult to be compared on account of the differences in imaging protocols, differences in histotype and clinical stage of tumor series included.

Analyzing the results, WB-MRI seems to be a valuable technique for $\mathrm{M}$ staging with equal or better performance than PET-CT, with advantages in detection of hepatic and pelvic lesions, bone and brain localizations. Although the diagnostic performance of each method should be assessed considering different possible bias (like imaging protocol, organ involved, lesion size), the major limiting factors of conventional WB-MRI, overcome by metabolic information provided by PET-CT, concern with the assessment of primary tumor extent (T-stage), differentiation between viable tumor from post-operative or adjacent normal tissue, especially when diagnosis is based on morphologic criteria alone and the evaluation of lymph-node status (N-stage) if morphological features and dimensional cut-off were used as the only pathological criteria [12,13].

Whereas the diagnostic capability of DWI in the identification of many primary tumors, distant metastasis and pathologically involved lymph node, showing restricted diffusion signal is known [13], the real impact of DWIBS technique remains to be determined on larger series.

Up to date, the diagnostic performance of DWIBS has been demonstrated in preliminary studies concerning with bone metastatic involvement for primary tumor that had the potential to diffuse to skeleton and for haematological disease (lymphoma and multiple myeloma) [14]. The role of DWIBS in the detection of extra-bone primary tumors has been demonstrated in a few experiences, with heterogeneous populations and low numbers of patients $[15,16]$. 


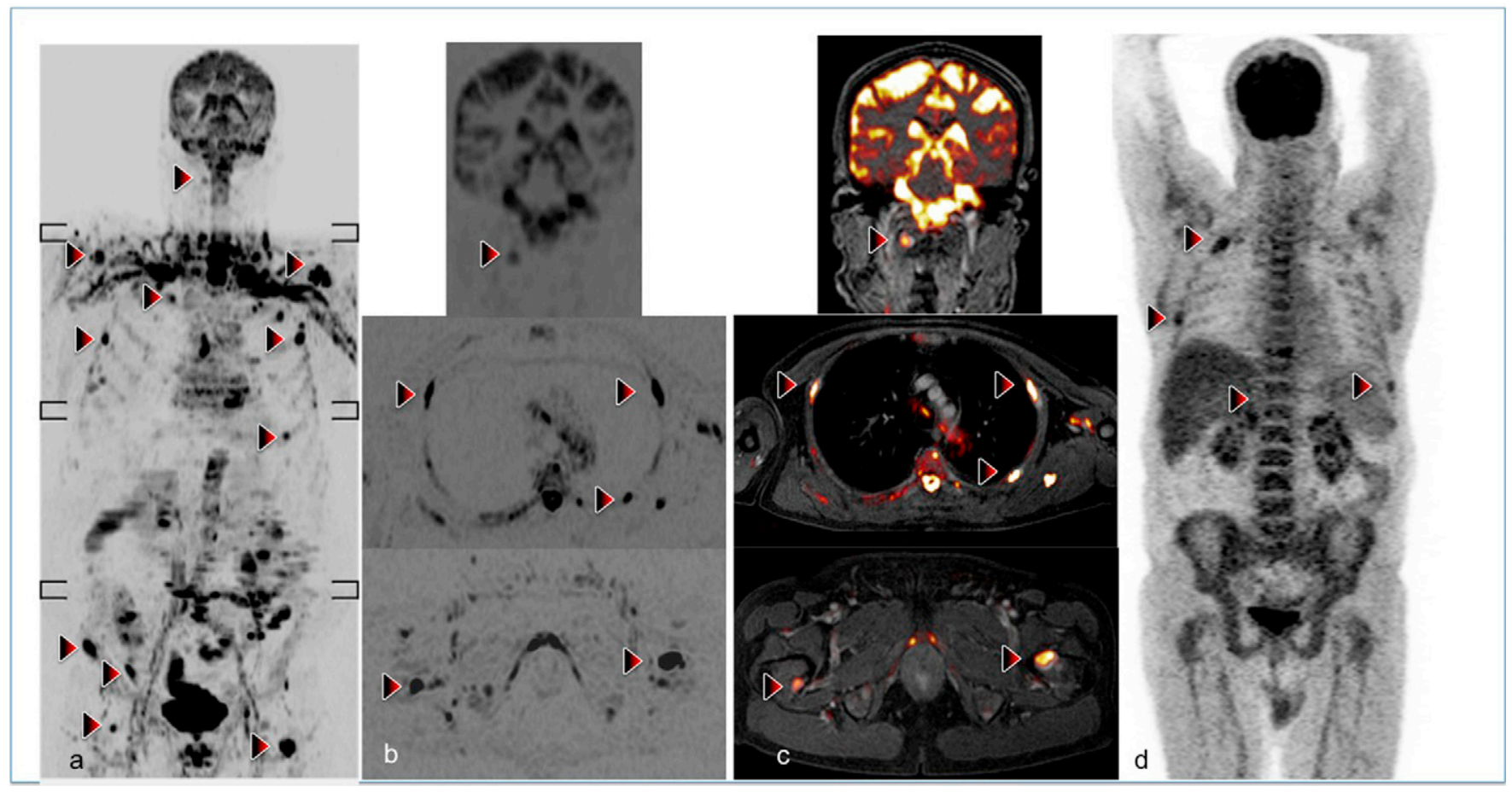

Fig. 7. 54 years-old patients with IgG multiple myeloma for 6 years showed extensive marrow replacement by plasma cell at bone marrow aspiration with high serum paraprotein level of $48 \mathrm{~g} / \mathrm{l}$. (a) coronal maximum intensity projection DWIBS highlights multiple focal areas with impeded diffusion at axial and limbs skeletal, consistent with extensive marrow packing. To note, as potential drawbacks, the presence of restricted diffusion from normal organs (es. small bowel, bladder, prostate, spinal cord, nerve roots) that may affect the diagnostic performance of DWIBS in detecting true malignant lesions. The use of DWIBS images (b) fused with 3D-CE-T1 at corresponding levels (c), may be used to confirm suspected positive lesions or to exclude normal findings with impeded diffusion; (d) coronal maximum intensity projection 18F-FDG PET-CT shows extensive but no specific slight tracer uptake at spine with lower numbers of FDG-avid lesions (arrowheads), compared with extensive involvement shown at DWIBS image. In this case, the combined use of 18F-FDG PET with CT (not shown) allows identifying extensive osteolitic pattern of disease with low grade of FDG uptake.

DWIBS imaging provides image with high contrast-to-noise ratio (CNR), leading to easy identification of small lesions during visual analysis and shortening reading time required to review the huge number of images provided by conventional WB-MRI. The main limitation of this technique concerns with sensitivity to artefacts determining signal loss for incoherent tissue motion (e.g. heart beats and diaphragm movements) and low anatomical resolution that may limit exact localization of a lesion, hampering the assessment of tumor infiltration into adjacent organs.

Furthermore, benign pathologies (cyst, abscess) or normal organ (brain, salivary glands, tonsils, spleen, gallbladder, small bowel, intestinal contents, colon, adrenal glands, prostate, testes, penis, endometrium, ovaries, spinal cord, peripheral nerves, lymph nodes, and bone marrow) may show impeded signal intensity on DWIBS images without evidence of disease status [17].

In our experience, these drawbacks may be overcome by combining DWIBS with standard morphological or contrast enhanced MR imaging (3D-CE-T1W) to provide anatomical information and to differentiate between malignant and normal tissue that may show abnormal signal intensity on DWIBS. To date, there is no consensus on which MR sequence should be used to improve diagnostic certainty of WB-MRI exam with DWIBS acquisition.

Tsushima et al. retrospectively evaluated 37 patients with abdomen malignancies and 73 patients, as control group, to assess the added value of fused DWI/T2W sequences in comparison with DWI and T2W sequence alone, viewed in blinded analysis as screening tool. Diagnostic performance (in terms of AUC) of fused DWI/T2w (0.904) was significantly higher than DWI $(0.720$; $p<0.01)$ and $\mathrm{T} 2 \mathrm{~W}(0.822 ; p<0.05)$ sequences considered separately. Specificity of fused DWI/T2W sequences $(81.9 \%)$ was higher than DWI (59\%; $p<0.001)$ and T2w (68.8\%; $p<0.01)$ images and sensitivity of fused images (89.5\%) was higher than that of DWI alone (72.4\%; $p<0.01$ ) [18].

In our experience, both sensitivity and specificity of DWIBS are improved by reviewing fusion images with 3D-CE-T1W and the improved specificity of DWIBS from 42 to $81.9 \%$ significantly contributes to increase AUC.

To our knowledge, no previous report verifies the diagnostic performance of DWIBS imaging fused with 3D-CE-T1W. As expected, many normal organs or benign findings ( $n=40 ; 1$ splenic haemangioma; 1 osteoporotic vertebral fracture; 1 vertebral haemangioma; 5 pancreatic cysts; 21 liver haemangiomas; 5 lymph nodes; 6 liver cysts) showed slight high-signal intensity in DWIBS. The absence of significant contrast enhancement or the typical pattern of enhancement revealed their benign nature. In only two cases (splenic haemangioma, vertebral haemangioma) with equivocal features also in fused images were resolved in consensus using morphologic data provided by $\mathrm{T} 1$ and $\mathrm{T} 2$ weighted images, included in our standard protocol. The use of high-contrast resolution sequences (3D-CE-T1W) allows to correctly assess anatomical localization of abnormal signal intensity (Fig. 5). Furthermore, evaluation of contrast enhancement or its pattern leads to useful identification of malignant vs. benign findings and avoids the high number of possible false-positive results provided by DWIBS (Fig. 6).

In the assessment of individual lesions, fused DWIBS/3D-CET1W images were statistically superior to DWIBS and 3D-CE-T1W, while the difference between DWIBS and 3D-CE-T1W did not reach statistical significance in our population. The use of DWIBS may increase the sensitivity of 3D-CE-T1W with potential role in identification of malignant lymph nodes without significant modification of morpho-dimensional parameters or primary lesions/distant 
metastasis overlooked on account the large amount of images to be review.

It is reported that the discrimination between malignant vs. benign lymph node is possible on account of qualitative criteria. Whereas normal lymph-nodes may show a relative high signal on DWIBS, metastatic lymph-nodes may have much more restricted diffusion related to their increased cellularity [19].

In our experience, 9 lymph nodes with slight high-signal intensity but no clear localization on DWIBS were correctly reclassified as malignant after reviewing fused images, regardless of their normal size at 3D-CE-T1W images in which faded contrast enhancement was demonstrated. Otherwise, in 2 patients with lung cancer and 1 patients with breast carcinoma (shown in Fig. 7), 4 equivocal lymph nodes ( 1 at inguinal site and 1 iliac lymph node for lung cancer patients; 2 axillaries lymph nodes for a breast cancer patient, respectively) suspicious for restricted diffusion were considered benign because of no reasonable localization according to their primary tumor in lung cancer patients and/or no suspicious features at 3D-CE-T1W images (no significant increase in size and no significant contrast-enhancement) in breast carcinoma patient. For both groups, 6 month follow-up imaging confirmed the benign nature.

The qualitative agreement between fused DWIBS/3D-CE-T1W and PET-CT images is encouraging and confirms the sensitivity and specificity of imaging based on fused DWIBS/3D-CE-T1W.

It remains challenging to demonstrate if the evaluation of ADC (not sampled in our experience) may be useful to clearly distinguish benign from malignant lymph nodes. However, recent studies showed not significant differences with overlapping of ADCs values between both types of lymph nodes in patients with abdominal and pelvic malignant disease $[19,20]$.

\section{Conclusion}

The availability of integrated morpho-functional data within a single exam, allow considering WB-MRI with DWIBS as an alternative imaging tool compared to conventional whole-body methods for the detection of primary tumor and distant metastasis or imaging follow-up in cancer patients.

PET-CT seems to be a more reliable method in identifying lymph-node status even if potential drawbacks may affect the diagnostic performance. Distinguishing role between malignant and non-malignant lymph-nodes should be investigated in larger series, but DWIBS may be considered a valuable technique for lymph-nodes identification. Even if the appropriate imaging protocol remains to be determined (T1W, T2W, T2W-STIR, 3D-CE-T1W), DWIBS requires to be reviewed side-by-side or in fused fashion with co-registered morphologic or contrast-enhanced images, used as anatomical map for localization and characterization of abnormal findings at the same site.

\section{Conflict of interest}

The authors state no conflict of interest.

\section{References}

[1] Antoch $\mathrm{G}$, Saoudi $\mathrm{N}$, Kuehl $\mathrm{H}$, et al. Accuracy of whole-body dual-modality fluorine-18-2-fluoro-2-deoxy-D-glucose positron emission tomography and computed tomography (FDG-PET/CT) for tumor staging in solid tumors: comparison with CT and PET. J Clin Oncol 2004;22(21):4357-68.

[2] Schmidt GP, Kramer H, Reiser MF, Glaser C. Whole-body magnetic resonance imaging and positron emission tomography-computed tomography in oncology. Top Magn Reson Imaging 2007;18(3):193-202.

[3] Koh DM, Collins DJ. Diffusion-weighted MRI in the body: applications and challenges in oncology. AJR 2007;188(6):1622-35.

[4] Schmidt GP, Reiser MF, Baur-Melnyk A. Whole-body MRI for the staging and follow-up of patients with metastasis. Eur J Radiol 2009;70(3): 393-400.

[5] Kwee TC, Takahara T, Ochiai R, et al. Diffusion-weighted whole-body imaging with background body signal suppression (DWIBS): features and potential applications in oncology. Eur Radiol 2008;18(9):1937-52.

[6] Fischer MA, Nanz D, Hany T, et al. Diagnostic accuracy of whole-body MRI/DWI image fusion for detection of malignant tumors: a comparison with PET-CT. Eur Radiol 2011;21(2):246-55.

[7] Blake MA, Singh A, Setty BN, et al. Pearls and pitfalls in interpretation of abdominal and pelvic PET-CT. Radiographics 2006;26(5):1335-53.

[8] Manenti G, Di Roma M, Mancino S, et al. Malignant renal neoplasms: correlation between ADC values and cellularity in diffusion weighted magnetic resonance imaging at 3 T. Radiol Med 2008;113(2):199-213.

[9] Padhani AR. Diffusion magnetic resonance imaging in cancer patient management. Semin Radiat Oncol 2011;21(2):119-40.

[10] Takahara T, Imai Y, Yamashita T, Yasuda S, Nasu S, Van Cauteren M. Diffusion weighted whole body imaging with background body signal suppression (DWIBS): technical improvement using free breathing. STIR and high resolution 3D display. Radiat Med 2004;22(4):275-82.

[11] Squillaci E, Manenti G, Mancino S, et al. Staging of colon cancer: wholebody MRI vs. whole-body PET-CT-initial clinical experience. Abdom Imaging 2008;33(6):676-88.

[12] Kwee TC, Takahara T, Ochiai R, et al. Complementary Roles of Whole-Body Diffusion-Weighted MRI and 18F-FDG PET: The State of the Art and Potential Applications. J Nucl Med 2010;51(10):1549-58.

[13] Nakanishi K, Kobayashi M, Nakaguchi K, et al., Whole-body MRI. for detecting metastatic bone tumor: diagnostic value of diffusion-weighted images. Magn Reson Med Sci 2007;6(3):147-55.

[14] Luciani A, Lin C, Beaussart P, Zerbib P, Haioun C, Rahmouni. Whole body functional MR imaging: hemato-oncologic applications. J Radiol 2010;91(3 (Pt. 2)):375-80.

[15] Komori T, Narabayashi I, Matsumura K, et al. 2-[Fluorine-18]-fluoro-2-deoxyD-glucose positron emission tomography/computed tomography versus whole-body diffusion-weighted MRI for detection of malignant lesions: initial experience. Ann Nucl Med 2007;21(4):209-15.

[16] Wang N, Zhang M, Sun T, et al. A comparative study: diffusion weighted whole body imaging with background signal suppression and hybrid Positron Emission Tomography on detecting lesion in oncologic clinics. Eur J Radiol 2011. April 6 [Epub ahead of print].

[17] Kwee TC, Takahara T, Ochiai R, et al. Whole-body diffusion-weighted magnetic resonance imaging. Eur J Radiol 2009;70(3):409-17.

[18] Tsushima Y, Takano A, Taketomi-Takahashi A, Endo K. Body diffusion-weighted MR imaging using high b-value for malignant tumor screening: usefulness and necessity of referring to T2-weighted images and creating fusion images. Acad Radiol 2007;188(4):643-50.

[19] Kim JK, Kim KA, Park BW, Kim N, Cho KS. Feasibility of diffusion weighted imaging in the differentiation of metastatic from non-metastatic lymph nodes: early experience. J Magn Reson Imaging 2008;28(3):714-9.

[20] Nakai G, Matsuki M, Inada Y, et al. Detection and evaluation of pelvic lymph nodes in patients with gynecologic malignancies using body diffusion-weighted magnetic resonance imaging. J Comput Assist Tomogr 2008;32(5):764-8. 